Document downloaded from:

http://hdl.handle.net/10251/67404

This paper must be cited as:

Serrano, X.; Escrivá-Escrivá, G. (2015). Simulation Model for Energy Integration of Distributed Resources in Buildings. IEEE Latin America Transactions. 13(1):166-171. doi:10.1109/TLA.2015.7040644.

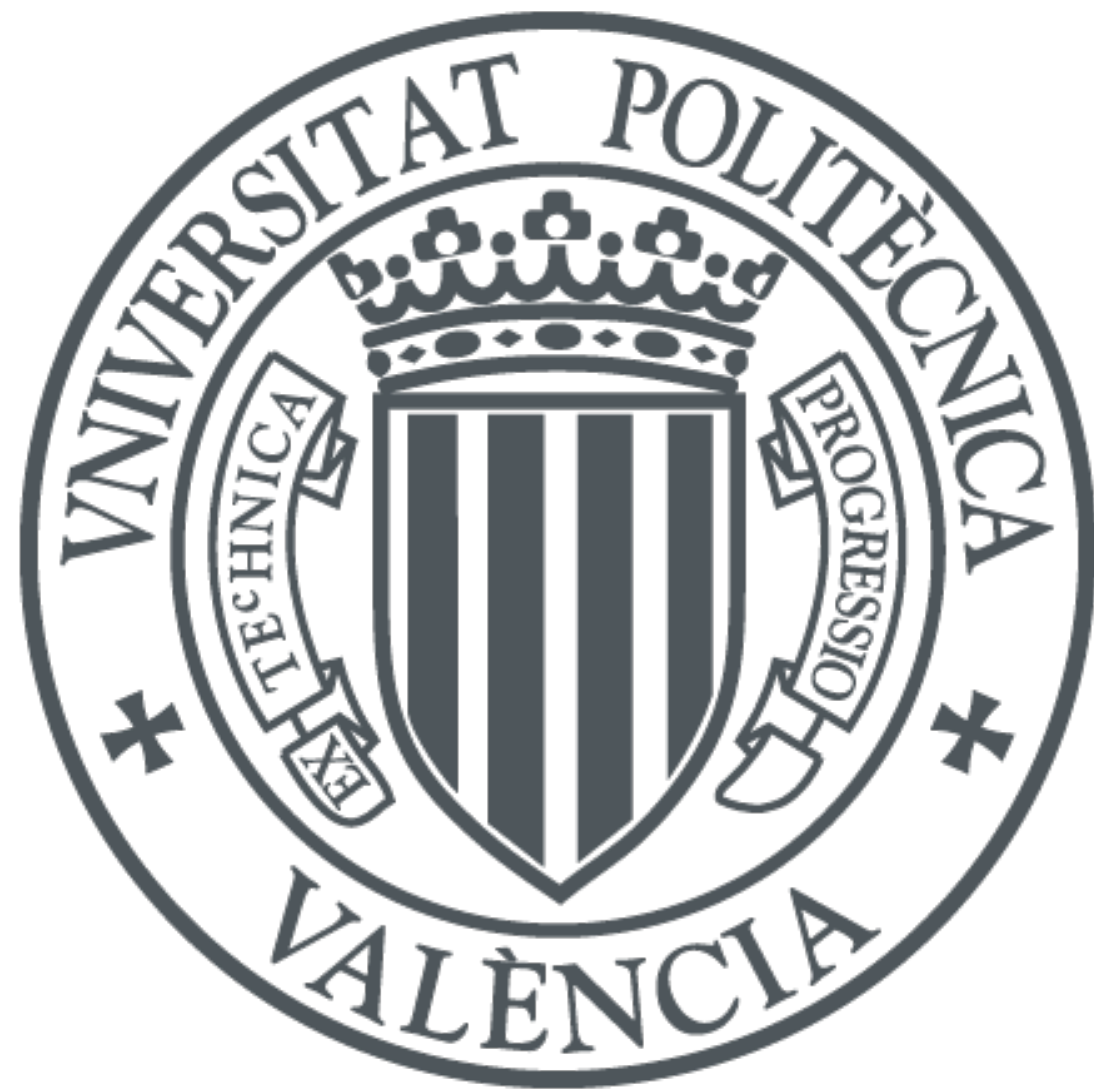

The final publication is available at

http://dx.doi.org/10.1109/TLA.2015.7040644

Copyright Institute of Electrical and Electronics Engineers (IEEE)

Additional Information

"(c) 2016 IEEE. Personal use of this material is permitted. Permission from IEEE must be obtained for all other users, including reprinting/ republishing this material for advertising or promotional purposes, creating new collective works for resale or redistribution to servers or lists, or reuse of any copyrighted components of this work in other works." 


\title{
Simulation Model for Energy Integration of Distributed Resources in Buildings
}

\author{
J.X. Serrano ${ }^{1}$, G. Escrivá ${ }^{2}$
}

\begin{abstract}
In the last few years, several problems have been evidenced in the electricity sector for example: increasing energy demand, overloaded power grids, transport losses in energy, resource shortages and greenhouse gas emissions in their generation. In response to these problems, several solutions have been proposed, one of them is the distributed generation with clean technologies, where each industry, building, housing, etc., is capable of generate energy to supply your own demand.
\end{abstract}

The objective of this research is to present a simulation model for the integration of distributed resources in buildings, that is able to predict the energy contribution of each technology in a distributed resource's system in a building, using as inputs: the local climate (solar radiation, wind speed, etc.,), building electricity demand, contracted electrical power, and the size of the available distributed resources. The main advantage of this model is that it allows simulations of various scenarios and in different parts of the world.

Keywords - distributed resources, energy integration, distribution generation, renewable energy

U na red eléctrica posee varios elementos, entre ellos están las líneas de trasmisión, transformadores, protecciones, los cuales están encargados de llevar la energía desde las Centrales de Generación CG hacia los consumidores, las CG están físicamente alejadas de las poblaciones razón por la cual la energía debe ser transportada largas distancias hasta que llega a un punto de consumo. Estas redes deben estar preparadas para suplir la creciente demanda existente por parte de los consumidores.

\section{INTRODUCCIÓN}

El transporte de energía siempre produce pérdidas, por ello se ha planteado un nuevo concepto de red eléctrica en la cual aparecen los Recursos Distribuidos RD, es decir fuentes de generación que se encuentran distribuidas a lo largo de la red y mucho más cercanas al consumidor [1], lo cual minimiza las pérdidas por transporte y hace al sistema más eficiente, estas ventajas se magnifican cuando la Generación Distribuida GD

\footnotetext{
${ }^{1}$ J. X. Serrano, Universidad Politécnica Salesiana (UPS), Cuenca, Ecuador, jserranog@ups.edu.ec

${ }^{2}$ G. Escrivá, Universidad Politécnica de Valencia (UPV), Valencia España, guieses@die.upv.es
}

proviene de fuentes de energía renovable FER ya que evitan emisiones de gases de efecto invernadero GEI.

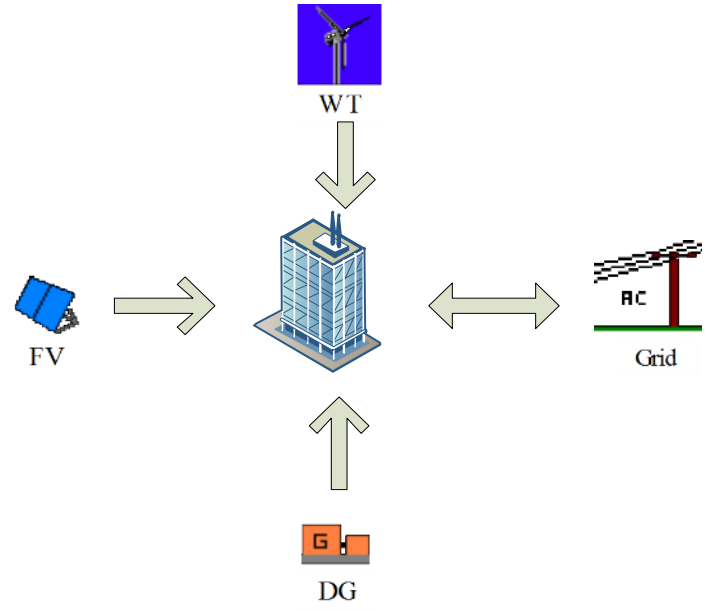

Figura 1. Integración de recursos distribuidos en edificios

\section{A. Recursos Distribuidos RD}

Los RD son elementos de generación de pequeña potencia (0-5MW) dispersos a lo largo de la red de distribución [2], esto consigue disminuir la sobrecarga en el transporte y distribución. Los RD pueden ser: motores de combustión interna alternativos, turbinas de gas, eólicas o hidráulicas, pilas de combustible, paneles fotovoltaicos, energía solar térmica, etc. [3].

Como ventajas de los RD se puede citar que evitan realizar grandes proyectos, permiten retrasar la ampliación de las líneas de transmisión y distribución de energía, reducen pérdidas, mejoran niveles de tensión, fiabilidad y calidad del sistema, se incorporan nuevas empresas y puestos de trabajo en el sector. Utilizan un gran aporte de energías limpias y su consiguiente bajo impacto ambiental. Además permiten el almacenamiento distribuido, el cual facilita el balance entre generación y consumo, ya que la GD renovable no puede ser regulada, de tal manera que momentos en los que existe un exceso de producción esta energía puede ser almacenada y por el contrario cuando existe un déficit en la producción la energía almacenada puede suplir la demanda. Los excedentes de energía pueden venderse a la compañía eléctrica [4], [7].

Existen desafíos en la implementación de los RD, la red de distribución no está diseñada para incluir GD y tampoco para soportar un flujo bi-direccional de energía. Los RD pueden 
interrumpir la operación de la red de distribución si no se consideran adecuadamente, un interruptor podría aislar una sección de la red de distribución por lo que el generador alimentaría la carga en "Isla" (Islanding) [10]. Existe complejidad en su control, desde el punto de vista de la calidad de la energía y del balance de potencia. Por lo general requieren de un dispositivo electrónico de potencia (rectificador e inversor) que convierta la tensión entregada a la utilizada por la red en términos de valor eficaz y de frecuencia [5] [6].

Es importante contribuir al estudio de la integración de los RD y las energías renovables ER a la red eléctrica convencional, todo aquello como un paso previo al desarrollo de las redes inteligentes (Smart Grids) SG [11]. Es necesario dimensionar correctamente los RD, de tal manera que se tomen las consideraciones técnicas adecuadamente [13], sin la necesidad de realizar una implementación física.

La utilidad del método de simulación propuesto en este trabajo se plantea ante la carencia de un software específico para cuantificar la contribución energética de recursos distribuidos en edificaciones en función de la demanda y el clima. La ventaja principal de disponer de los datos climatológicos de muchos lugares a lo largo del planeta, es que permite la simulación de sistemas energéticos que dependen del clima (radiación solar, viento, temperaturas, etc.,), haciendo posible que se puedan evaluar las diferencias de implementar un mismo sistema energético en diferentes locaciones del mundo.

El trabajo está estructurado en 4 secciones, en la primera se trata una breve revisión del estado del arte, destacándose al final la justificación del modelo desarrollado, en la sección 2 y 3 se explica la metodología utilizada para la implementación del modelo tomando como referencia normativas y políticas europeas, su funcionamiento y potencialidades y finalmente se muestran las conclusiones.

Para realizar el estudio y simulación se ha elegido un edificio (8E Y 8F) de la Ciudad Politécnica de la Innovación (CPI) dentro de la Universidad Politécnica de Valencia (UPV), ubicado en Valencia, España.

\section{METODOLOGÍA DEL MODELO DE SIMULACIÓN}

El modelo de simulación para la integración energética de recursos distribuidos en edificios se basa en ciertos datos que pueden ser recopilados y otros parámetros que deben ser dimensionados. Estos datos y parámetros son los siguientes: la demanda de energía eléctrica del edificio, clima del lugar (radiación solar, temperatura, velocidad del viento), potencia eléctrica contratada en el edificio, RD (energía solar, eólica, grupo electrógeno, banco de baterías) disponibles y su dimensión. El objetivo del simulador es determinar la contribución energética de cada tecnología en el sistema y predecir la interrelación entre consumo y generación.

Para la implementación del modelo de simulación se ha escogido el software TRNSYS debido a su gran versatilidad, es un software de código abierto que permite introducir los datos climatológicos de un año entero de la zona en estudio [12], así como también la demanda energética, contiene modelos muy confiables de elementos de los sistemas energéticos que se requieren, permite realizar balances anuales, interanuales o diarios, estudios paramétricos que permiten evaluar objetivamente optimizaciones por lo cual es ideal para realizar estudios de viabilidad. Es una herramienta flexible que se adapta a cualquier tipo de cambios que requieren investigadores y profesionales de la comunidad de la simulación de la energía, esto ha hecho que importantes entidades a nivel mundial continúen utilizando este programa por ejemplo la NASA, National Renewable Energy Lab (USA), BMW, Electricité de France, entre otros [8].

\section{A. DESCRIPCIÓN DEL MODELO}

El modelo se compone de distintos bloques orientados a realizar determinadas tareas, en la Fig. 2 se muestra el diagrama de bloques.

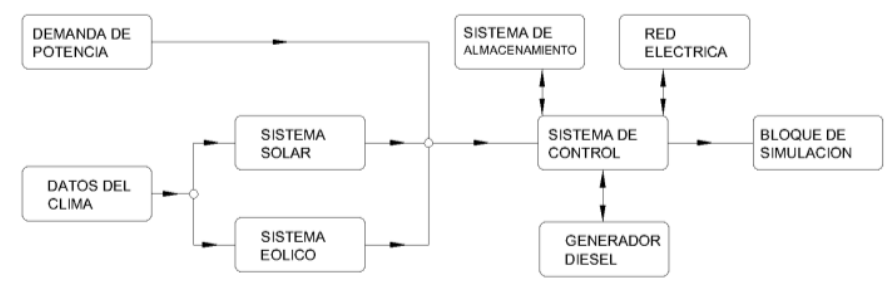

Figura 2. Diagrama de bloques del modelo

La demanda de energía eléctrica ha sido obtenida de la curva cuarta horaria de uno de los edificios de la Universidad Politécnica de Valencia (UPV) de todo el año 2012, el modelo lee esta información directamente, desde una hoja electrónica.

El bloque "Datos del Clima" adquiere los datos climáticos dentro de un periodo de simulación, estos datos climatológicos se encuentran en formato TMY2, y se eligen de acuerdo a la localidad en la cual se realiza la simulación [8]. Este bloque es capaz de presentar datos, entre ellos: temperatura ambiente $\left[{ }^{\circ} \mathrm{C}\right]$, humedad relativa $[\%]$, velocidad $[\mathrm{m} / \mathrm{s}]$ y dirección del viento [grados], presión atmosférica $[\mathrm{Pa}]$, radiación solar total , directa y difusa sobre una superficie inclinada [kJ/hr.m^2], etc., estos datos sirven de entrada para los siguientes bloques, tanto para el sistema de generación solar, como para el sistema de generación eólico, ya que estos bloques requieren de estos datos para estimar la cantidad de energía que puede ser producida en un sistema fotovoltaico PV o eólico.

El bloque "sistema solar" es un sistema PV que básicamente requiere conocer las características y número de los paneles, inclinación y azimut. En el bloque del sistema eólico, se especifica al simulador, el tipo de aerogenerador y su potencia. 
En función de los datos climáticos, básicamente radiación solar y velocidad del viento y de la dimensión de los sistemas tanto solar como eólico se obtendrá una energía total generada de origen renovable. Esta energía total generada y la demanda ingresan como variables al bloque del sistema de control, para que éste tome las decisiones de qué sistema aportará energía, ya sea el de almacenamiento de energía (baterías), el grupo electrógeno (generador diesel) o la red eléctrica convencional.

El modelo de simulación presenta variables de salida, las cuales son el objeto de la simulación, estas se grafican clasificadas en tres bloques de visualización. Referirse a la Tabla I.

TABLA I

BLOQUES DE VISUALIZACIÓN DEL MODELO DE SIMULACIÓN

\begin{tabular}{|c|c|c|c|}
\hline Bloque & Magnitud & Unidad & Curvas \\
\hline 1 & Potencia & {$[\mathrm{kW}]$} & $\begin{array}{l}\text { Sistema Fotovoltaico } \\
\text { Sistema Eólico } \\
\text { Generador Diesel } \\
\text { Red Eléctrica } \\
\text { Convencional } \\
\text { Baterías }\end{array}$ \\
\hline \multirow{5}{*}{2} & \multirow{5}{*}{ Energía } & \multirow{5}{*}[\mathrm{kWh}]{} & Sistema Fotovoltaico \\
\hline & & & Sistema Eólico \\
\hline & & & Generador Diesel \\
\hline & & & $\begin{array}{l}\text { Red Eléctrica } \\
\text { Convencional } \\
\text { Baterías }\end{array}$ \\
\hline & & & Demanda \\
\hline \multirow[b]{2}{*}{3} & \multirow[b]{2}{*}{ Energía } & \multirow[b]{2}{*}[\mathrm{kWh}]{} & Energía Almacenada \\
\hline & & & Energía Entregada a Red \\
\hline
\end{tabular}

En el primer bloque se encuentran graficadas las curvas de potencia en [kW], como se muestra en la Fig. 3.

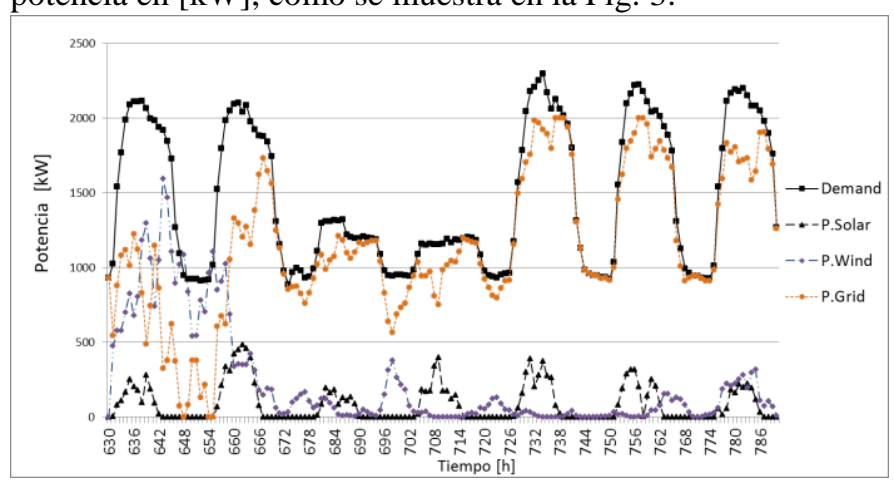

Figura 3. Visualización de potencias

La Fig. 4 muestra el segundo bloque de visualización, la energía aportada [kWh] de cada uno de los sistemas de manera acumulada, es decir, que a la hora final de la simulación se observa cuánta energía en total, ha aportado cada tecnología a lo largo del periodo de simulación.

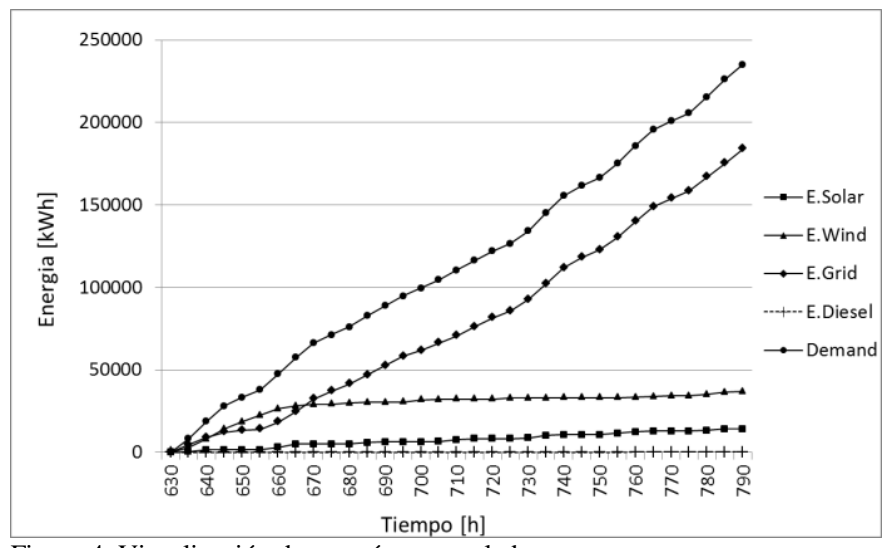

Figura 4. Visualización de energías acumuladas

En el tercer bloque de visualización se grafica la energía almacenada cuando se haya seleccionado el modo de simulación de almacenamiento de energía. En la Fig. 5 se ve que las baterías reciben energía y luego la entregan hasta cuando ésta llega a cero.

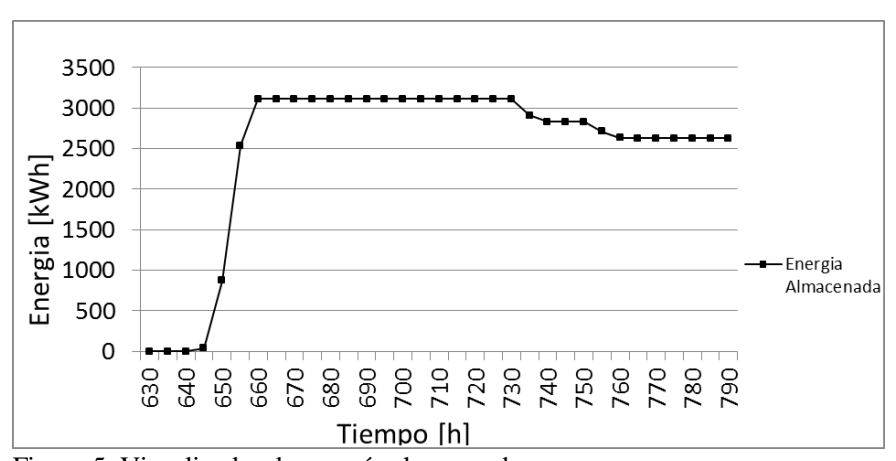

Figura 5. Visualizador de energía almacenada

Cuando se seleccione la opción de no almacenamiento de energía, es decir entregar energía a red, al tiempo final de la simulación la curva contabiliza la cantidad total de energía que ha sido entregada a red, tal como muestra la Fig. 6.

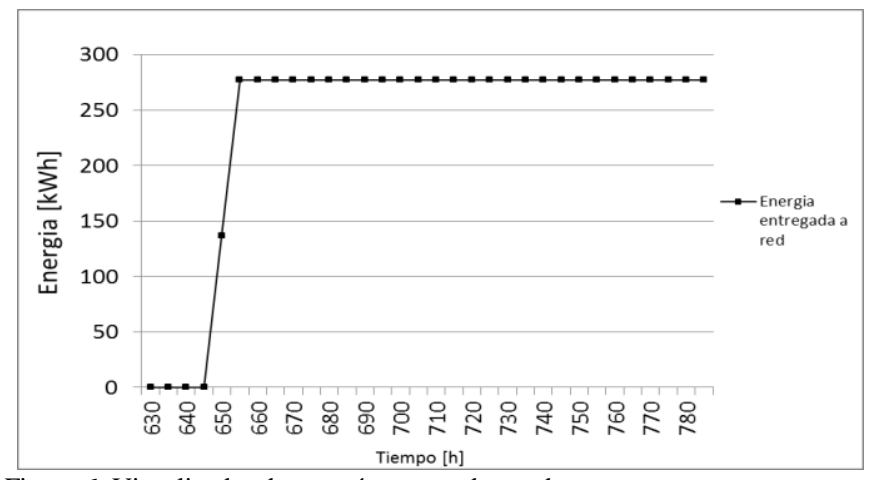

Figura 6. Visualizador de energía entregada a red

Todas estas variables son almacenadas automáticamente en una hoja electrónica. 


\section{B. Flujo de Energía del Modelo de Simulación}

Los bloques de generación eólica y solar aportan energía al sistema, siempre supervisados por un bloque de control. Las baterías pueden absorber o entregar energía en función de las necesidades del sistema. El grupo electrógeno exclusivamente entrega energía y sólo cuando sea necesario, el funcionamiento de estos bloques puede ser ajustado de acuerdo a modos de funcionamiento que pueden ser seleccionados en el modelo de simulación [9].

El modelo de simulación permite tomar o entregar potencia de la red, de acuerdo al modo de funcionamiento seleccionado. La demanda energética es abastecida por todo el conjunto de dispositivos que existen en el sistema, pero siempre coordinados por un bloque de control, el cual es el encargado de obtener el máximo provecho de los elementos del sistema.

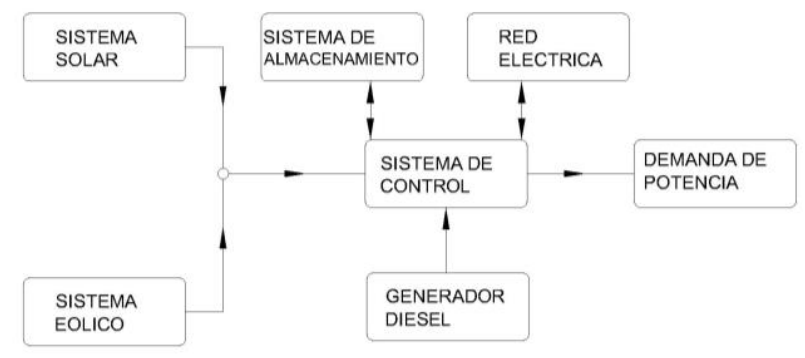

Figura 7. Diagrama de Flujo de Energía

\section{Diagrama de flujo del modelo de simulación}

En la Fig. 8 se muestra un diagrama de flujo que se ajusta de la manera más cercana posible al funcionamiento del programa debido a que TRNSYS utiliza una programación por bloques. En este diagrama se puede apreciar la filosofía del modelo de simulación.

El sistema inicia con la obtención de datos, tanto climatológicos, como de la demanda. Una vez obtenida la información del clima y en base a la dimensión del sistema solar y eólico, el software calcula la producción de energía de origen renovable total, la cual es el resultado de la suma de la producción del sistema solar más la del sistema eólico; a continuación se tiene un elemento de decisión en el cual se comparan los valores de demanda y de generación renovable. Si la demanda es menor a la generación renovable se cubre directamente con la energía renovable, en este caso, si no existe exceso de energía, el ciclo de simulación termina, esto es poco probable que ocurra en la realidad ya que es difícil que la generación cuasi aleatoria de origen solar y del viento se iguale a la demanda, en cambio la opción que exista un exceso de generación ocurre pocas veces, por lo que el sistema verifica si existen baterías para cargarlas, en el caso contrario se entrega la energía excedente a red.

Cuando la demanda es mayor que la generación renovable lo cual es muy frecuente, el sistema calcula el déficit, es decir cuanta potencia es necesaria para satisfacer la demanda. Si el déficit de potencia es menor que la potencia contratada, este déficit se cubre con potencia de red, sin embargo si el déficit es mayor a la potencia contratada el sistema lleva a cabo acciones que permitan impedir que esto ocurra.

En el caso de que el déficit sea mayor a la potencia contratada, el sistema verifica si las baterías tienen energía almacenada, en el caso afirmativo se usa esta energía y se vuelve a comparar el déficit con la potencia contratada, si las baterías llegan al punto crítico de descarga, no estaban cargadas o su energía no es suficiente para cubrir con el exceso de demanda, el controlador del sistema indica al generador diesel que se ponga en marcha para cubrir el déficit restante.

El generador diesel no puede generar por encima de su potencia nominal, el modelo de simulación verifica si la potencia que se requiere que el generador diesel genere es mayor que su potencia nominal, en ese caso, el generador genera a la potencia nominal y el déficit restante es cubierto por la red, en este caso inevitablemente se excede la potencia contratada. Si la potencia requerida es menor a la potencia nominal el generador diesel solamente generará lo necesario.

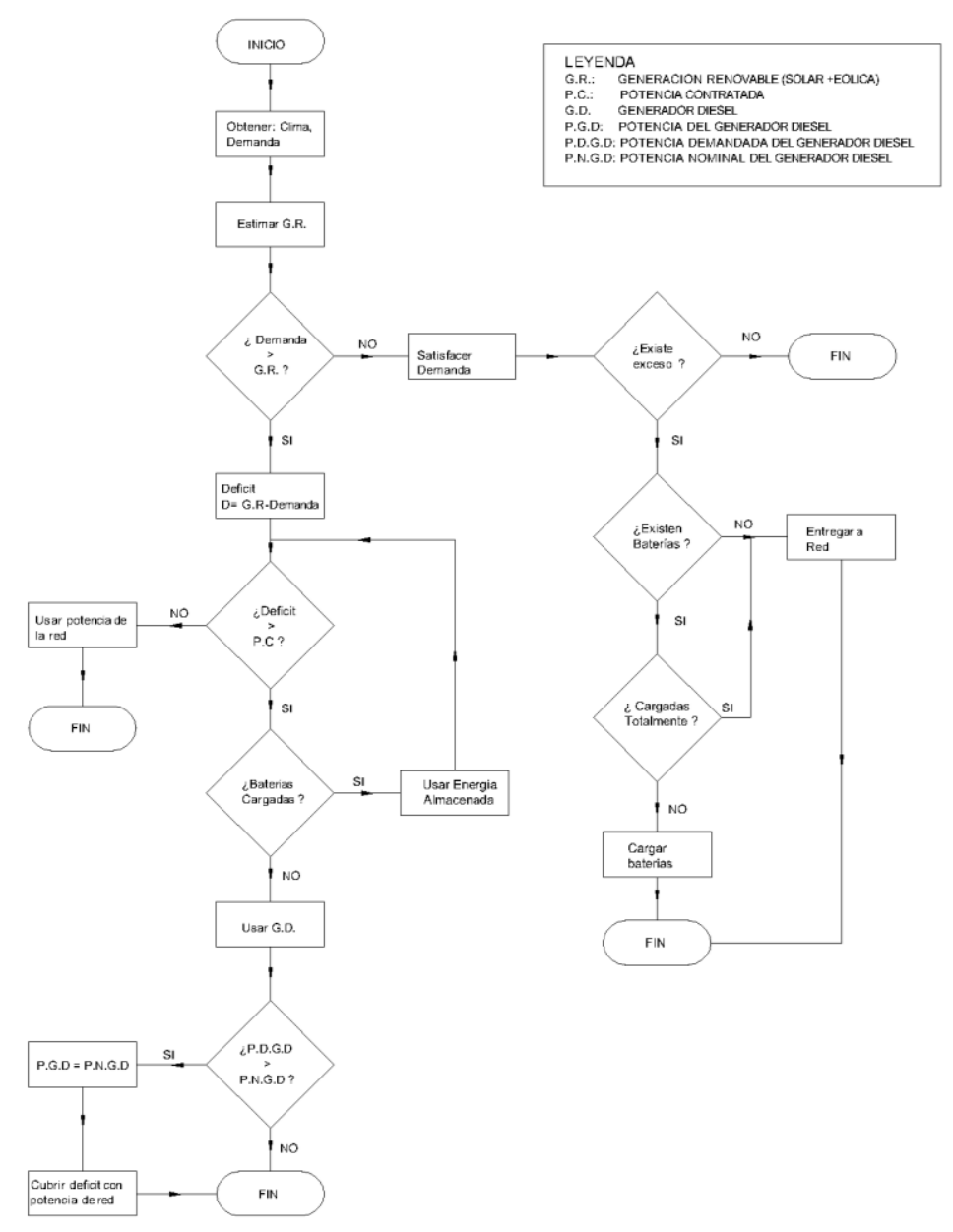

Figura 8. Diagrama de Flujo de del Modelo de Simulación 


\section{Modos de funcionamiento del modelo de simulación}

El modelo de simulación permite seleccionar ciertos modos simulación tales como:

1. Entregar energía sobrante a la red: El sistema no almacena energía cuando la generación renovable excede a la demanda, sino que la inyecta directamente a la red.

2. Almacenar energía sobrante en baterías: La energía excedente se almacena en baterías y no se entrega a red.

3. Utilizar la energía almacenada en baterías: Cuando se almacena la energía en baterías pueden existir dos criterios; el primero es dejar esa energía almacenada para utilizarla cuando exista algún fallo (de red o de los sistemas internos del edificio) o la segunda opción es utilizarla cuando la potencia de red que se está utilizando llegue a superar el valor de la potencia contratada.

4. Generación de un grupo electrógeno: Se puede activar su generación cuando la potencia de red que se está utilizando llegue a superar el valor de la potencia contratada y no exista energía almacenada en baterías.

5. Habilitar el uso del sistema solar de generación: Para efectos de simulación se puede habilitar o deshabilitar el uso de la energía solar.

6. Habilitar el uso de un aerogenerador: De la misma manera que el caso anterior se puede habilitar o deshabilitar el uso de uno o varios aerogeneradores.

\section{POTENCIALIDADES DEL MODELO DE SIMULACIÓN}

Se pueden evaluar diferentes tipos de escenarios energéticos de manera muy confiable. Es posible realizar un análisis de periodos cortos de tiempo; se presentan las curvas de simulación para dos semanas en verano con el objeto de observar cómo interactúan entre sí los diferentes componentes del sistema. En la Fig. 9 se puede ver la curva de la demanda, la energía solar y la energía eólica; la generación de origen renovable no sobrepasa la demanda.

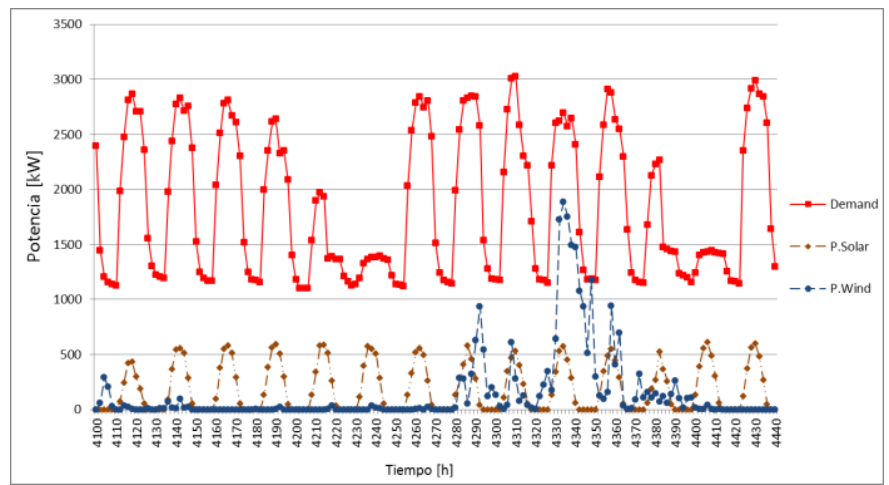

Figura 9. Demanda vs energía solar y eólica
En el mismo caso se observa que un generador diesel ha sido utilizado para evitar que la potencia utilizada de red, exceda la contratada $(2000 \mathrm{~kW})$, sin embargo cuando el generador diesel no puede generar a más de su potencia nominal $(400 \mathrm{~kW})$ inevitablemente la utilización de la potencia de red excede la contratada $(30,6 \mathrm{~kW}$ en la hora $4118,35,3 \mathrm{~kW}$ a las $4268 \mathrm{~h}$ y $175,8 \mathrm{~kW}$ en la hora 4434$)$, tal como se ve en la Fig. 10.

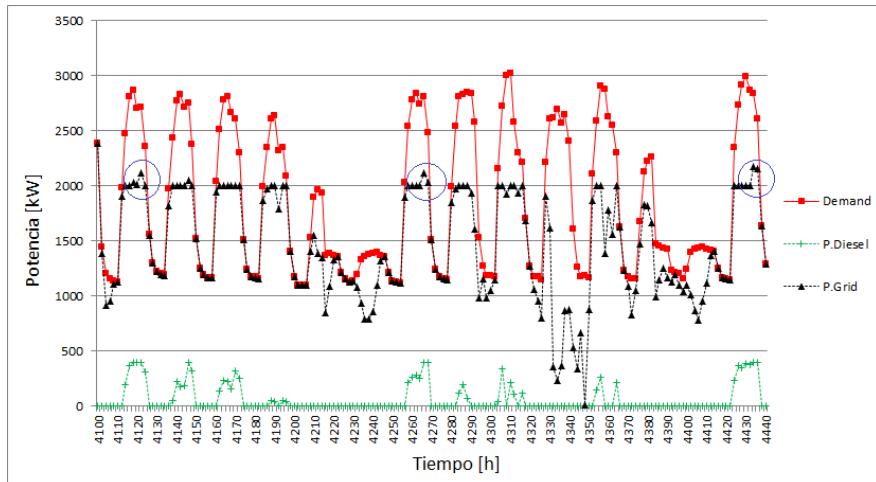

Figura 10. Excesos en la potencia de red

En este tipo de análisis es posible ver cómo interactúan las diversas tecnologías en el sistema de alimentación, por ejemplo, se ve que la utilización de la potencia de red disminuye considerablemente debido al uso de otros sistemas, permitiendo establecer un nuevo valor de potencia contratada, con sus respectivos ahorros económicos.

Se puede realizar un análisis del balance energético anual, obteniendo la cantidad de energía que aporta cada sistema e incluso la red, de tal manera que se pueda decidir anticipadamente la implementación de un sistema energético en función de su costo, producción anual y emplazamiento, permitiendo también cuantificar las emisiones de $\mathrm{CO} 2$ evitadas.

Se puede obtener el balance anual en la utilización de energías, es interesante analizar el aporte de cada tecnología en la generación de energía de todo el sistema, de la misma manera la cantidad de energía aportada por la red.

Es posible cuantificar la energía que se entregaría a red a lo largo del año de simulación (Fig. 11), cuyo valor final representa la cantidad total de energía que se ha entregado a red en todo el año, en este caso $31736 \mathrm{kWh}$.

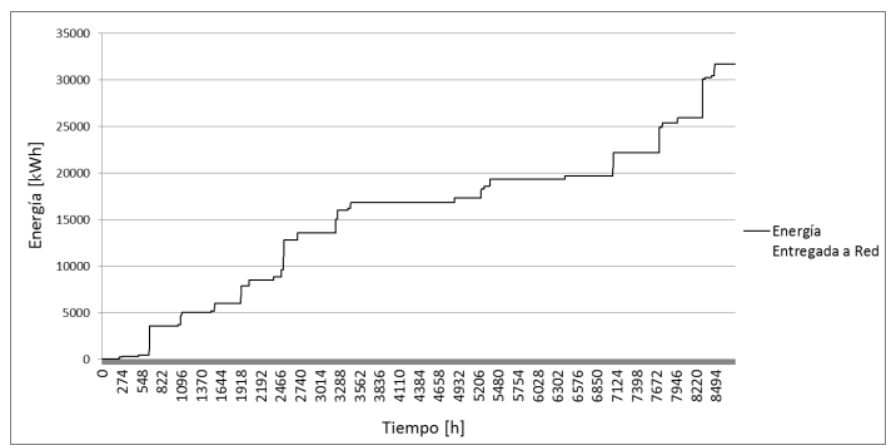

Figura 11. Energía Entregada a red 


\section{CONCLUSIONES}

El modelo de simulación permite cuantificar la cantidad de energía que puede producir, entregar a red o almacenar un conjunto de sistemas (eólico, solar, red, baterías, generador diesel), en función de sus características y dimensiones y de la localidad en la que se encuentren.

El sistema es capaz de entregar curvas de potencia de cada elemento de un sistema energético, con la curva de potencia de red se puede realizar un estudio de optimización de la potencia contratada de red.

La curva de potencia almacenada por el sistema permite dimensionar de manera óptima el banco de baterías o un sistema similar.

Las curvas de energía permiten realizar balances diarios, semanales, mensuales o anuales de energía, permitiendo efectuar análisis de factibilidad técnica, ambiental o económica ya que se pueden calcular fácilmente costos, emisiones de $\mathrm{CO} 2$ de cada una de las tecnologías implicadas.

La utilidad del método de simulación propuesto en este trabajo se plantea ante la carencia de un software específico para cuantificar la contribución energética de recursos distribuidos en edificaciones en función de la demanda y el clima.

Se pueden plantear trabajos futuros para cuantificar la contribución de recursos distribuidos, así como la reducción de emisiones de $\mathrm{CO}_{2}$ en localidades y edificaciones específicas.

\section{REFERENCIAS}

[1]D. Moskovitz, Profits and Progress Through Distributed Re-sources; published by the Regulatory Assistance Project, Maine, USA, also available at: http: //www.rapmaine.org/distribution.html, 2000

[2] Thomas Ackermann, Go"ran Andersson, Lennart Soder, "Distributed generation: a definition" http: www.elsevier.com/, 2000.

[3] International Energy Agency, Enhancing the Market Deploy-ment of Energy Technology: a Survey of Eight Technologies, Paris, 1997.

[4] Observatorio Industrial del Sector de la Electrónica, Tecnologías de la Información y Telecomunicaciones "Smart Grids y la Evolución de la Red Eléctrica", 2.2 Concepto de Red Inteligente, Centros Tecnológicos de España, 2011

[5] James Paul Valencia Quintero. "GENERACIÓN DISTRIBUIDA: DEMOCRATIZACIÓN DE LA ENERGÍA ELÉCTRICA", Universidad Nacional de Colombia, Bogotá, D.C., 2008.

[6] Integrating Distributed Resources into Electric Utility Distribution Systems: EPRI White Paper, EPRI, Palo Alto, CA: 1004061, 2001.

[7] Badrul H. Chowdhury, Chung-Li Tseng, EDITORIAL "Distributed Energy Resources: Issues and Challenges" University Of Missoury,
JOURNAL OF ENERGY ENGINEERING (C) ASCE pages 109 - 110, September 2007

[8]TRNSYS, Transient System Simulation Tool, www.trnsys.com, 2013.

[9] Isabel Bjork, "ENCOURAGING RENEWABLE ENERGY DEVELOPMENT", Handbook for International Energy Regulators , USAID, NARUC, 2011. Chapter II, page 23.

[10]Tantimaporn, T.; Jiyajan, S.; Payakkarueng, S., "Microgrid islanding operation experience," Electricity Distribution (CIRED 2013), 22nd International Conference and Exhibition on , vol., no., pp.1,3, 10-13 June 213

[11] Lakshminarayana, S.; Quek, T.Q.S.; Poor, H.V., "Combining cooperation and storage for the integration of renewable energy in smart grids," Computer Communications Workshops (INFOCOM WKSHPS), 2014 IEEE Conference on , vol., no., pp.622,627, April 27 2014-May 22014

[12] Abdalla, N., "Validated TRNSYS model for solar assisted space heating system," Applications of Information Technology to Renewable Energy Processes and Systems (IT-DREPS), 2013 1st International Conference \& Exhibition on the , vol., no., pp.139,143, 29-31 May 2013

[13] C. Ibarlucea, C. Coyos, J. Vaschetti, J. C. Goméz, "Distributed Generation: impact on Protections and Power Quality", IEEE LATIN AMERICA TRANSACTIONS, Vol. 11, No. 1, pp. 460-465, Feb. 2013.

Xavier Serrano Guerero nació en Cuenca, Ecuador en 1984. Ingeniero

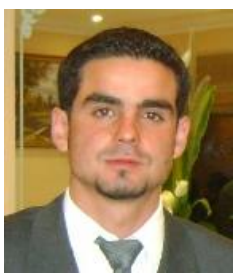
Electrónico en la Universidad Politécnica Salesiana, Cuenca, Ecuador 2008; Máster en Tecnología Energética para el Desarrollo Sostenible de la Universidad Politécnica de Valencia, España, 2012; Docente de la Univerisidad Politécnica Salesiana, Cuenca, Ecuador.

Guillermo Escrivá Escrivá recibió su grado de $\mathrm{PhD}$ en la Universitat Politècnica de València, es docente en la Escuela Técnica Superior de Ingenieros Industriales e investigador del Instituto de Ingeniería Energética en la Ciudad Politécnica de Innovación en la Universitat Politècnica de València, España. 